\title{
Genetic Variant rs755622 Regulates Expression of the Multiple Sclerosis Severity Modifier D-Dopachrome Tautomerase in a Sex-Specific Way
}

\author{
Zhijie Han, ${ }^{1}$ Jiaojiao Qu, ${ }^{2}$ Jiehong Zhao, ${ }^{3}$ and Xiao Zou $\mathbb{D}^{2}$ \\ ${ }^{1}$ Innovative Drug Research and Bioinformatics Group, School of Pharmaceutical Sciences, Chongqing University, Chongqing, \\ 401331, China \\ ${ }^{2}$ Institute of Fungus Resources, College of Life Sciences, Guizhou University, Guiyang, 550025, China \\ ${ }^{3}$ College of Pharmacy, Guiyang University of Chinese Medicine, Guian New Area, 550025, China
}

Correspondence should be addressed to Xiao Zou; xzou@gzu.edu.cn

Received 3 May 2018; Accepted 8 July 2018; Published 24 July 2018

Academic Editor: Marija Mostarica-Stojković

Copyright (C) 2018 Zhijie Han et al. This is an open access article distributed under the Creative Commons Attribution License, which permits unrestricted use, distribution, and reproduction in any medium, provided the original work is properly cited.

\begin{abstract}
Multiple sclerosis (MS) is a sex-specific autoimmune disease involving central nervous system. Previous studies determined that macrophage migration inhibitory factor (MIF) and its homologue D-dopachrome tautomerase (DDT) sex-specifically affect MS progression. Moreover, other studies reported that rs755622 polymorphism in promoter region of MIF gene is associated with risk of MS and affects the promoter activity to regulate MIF expression in a sex-specific way. Given that MIF and DDT share a part of promoter sequence, we surmise that rs755622 can also regulate DDT expression in a sex-specific way. However, this has not yet been studied. Here, we used five large-scale expression quantitative trait loci (eQTLs) and two RNA-seq datasets from brain and blood to assess the potential influence of rs755622 variant on expression of DDT in different genders by the linear regression and differential expression analysis. The results show that the minor allele frequency of rs755622 and expression of DDT are significantly increased in males for MS subjects and this minor allele variant can significantly upregulate $D D T$ expression for males but not females, which suggests that the regulation of DDT expression level by rs755622 can affect MS progression in males. These findings further support and expand conclusions of previous studies and may help to better understand the mechanisms of MS.
\end{abstract}

\section{Introduction}

Multiple sclerosis is one of the most common immunemediated diseases involving central nervous system and presents the sex-specific rates of morbidity [1-3]. According to the report provided by Atlas of MS in 2013, the number of individuals affected by MS is about 2.3 million [4]. Previous Genome-wide association studies (GWAS) uncovered that about 200 variants in the human genome are associated with MS and they mainly affect immunological process related genes and lie in their regulatory regions $[5,6]$.

An immunoregulatory cytokine, macrophage migration inhibitory factor (MIF), plays an important role in the modulation of macrophages and microglia immune response and is associated with autoimmune and inflammation-related diseases including MS [7-11]. D-dopachrome tautomerase
(DDT, also called MIF-2) is highly homologous to MIF and has a similar structure and functions as it [12-14]. Moreover, the promoter regions of $M I F$ and $D D T$ gene share sequences which contain some transcription factor binding sites [13, 14]. A recent study found that $D D T$ is a sex-specific disease modifier for MS, and its high expression can promote MS progression in males but not females [15].

The single nucleotide polymorphism (SNP) rs755622 (G $>\mathrm{C}$ ) lies in the promoter region of MIF, and previous studies showed that it is significantly associated with some immunemediated diseases including MS [16, 17]. And then, this recent study further indicated a significant sex difference in the association between rs755622 polymorphism and MS [15]. Moreover, previous studies determined that rs755622 polymorphism affects the activity of MIF promoter and regulates MIF gene expression [18-20]. Given the relationship between 
$M I F$ and DDT, we surmise that rs755622 polymorphism can also affect the expression of DDT. However, it is still unclear whether and how rs755622 polymorphism regulates DDT expression and the resulting impact on MS in different genders.

Evidence showed that regulating gene expression is an important class of the biological functions of the genetic variants [21-30], and the expression quantitative trait loci (eQTL) analysis is an effective method to discover the correlations between genetic variants and quantitative changes in gene expression [21, 22, 24, 25, 27, 31-33]. Therefore, in this study, we first selected five large-scale expression quantitative trait loci (eQTLs) datasets to assess the potential influence of rs755622 variant on expression of DDT in normal brain tissues and blood by a linear regression analysis. And then, we further investigated how the rs755622 polymorphism affects DDT expression in brain and blood of the MS patients using two RNA-seq datasets. Finally, we performed the differential expression analysis of DDT between genders and explored whether there is a sex-specific regulation of $D D T$ expression level by the rs755622 polymorphism.

\section{Methods}

2.1. eQTL Analysis Using Five Large-Scale Datasets. To validate the effect of rs755622 polymorphism on DDT expression level, we selected five large-scale eQTL datasets which primarily consist of the European ancestry individuals without MS diagnosis [34-38]. In particular, 10 brain regions (cerebellar cortex, frontal cortex, hippocampus, medulla, occipital cortex, putamen, substantia nigra, temporal cortex, thalamus, and intralobular white matter) of 134 individuals are included in Braineac [34]; 13 brain regions (amygdala, anterior cingulate cortex, caudate, cerebellar hemisphere, cerebellum, cortex, frontal cortex, hippocampus, hypothalamus, nucleus accumbens, putamen, spinal cord, and substantia nigra) of 1,497 individuals are included in GTEx [35]; the blood of $369,2,765,2,116$, and 5,257 individuals is included in GTEx [35], CAGE [36], BIOS QTL Browser [37], and FHS_eQTL [38], respectively. Then, we selected the rs755622 genotype and DDT expression data from Braineac and conducted the eQTL analysis to assess the influence of rs755622 variant on expression of $D D T$ by the R package 'Matrix eQTL', which is based on a linear regression model with the parameters, gender and age, as the covariates [39]. Finally, we analyzed the eQTL results for rs755622 (including beta and P values) from the other four datasets.

2.2. eQTL Analysis of MS Subjects Using the RNA-Seq Data. To further investigate the regulation of DDT expression by rs755622 polymorphism in MS patients, we first selected the RNA-seq data from 2 GEO datasets, GSE100297 (singleend reads produced by the Illumina HiSeq 3000) [40], and GSE66573 (paired-end reads produced by the Illumina HiSeq 2500) [41], which include the brain (optic chiasm) and blood samples of 5 and $6 \mathrm{MS}$ patients, respectively. We next mapped these sequences to human reference genome (hg19) and calculated the transcript per million (TPM) values to measure DDT expression level in each of the MS patients using the Kallisto software, which is a quantification tool of transcript abundance based on RNA-seq data [42]. Then, these RNA-seq data were reused to detect the genotype of rs755622 polymorphism in each of the MS patients. This process includes the following: (1) the sequence reads are aligned to reference genome (hg19) using BWA software with the default parameter settings [43] and (2) SNPs are called on these aligned reads using SAMtools software with the default parameter settings (100 read depth) [44]. For the called genotype of rs755622 polymorphism, a Hardy-Weinberg Equilibrium (HWE) test based on a noncontinuity correction chi-squared method with the significance level $P<0.05$ was performed using the $\mathrm{R}$ package 'Genetics' (https://cran.r-project.org/web/packages/ genetics/index.html). Finally, we used the DDT expression and rs755622 polymorphism genotyping data of these MS patients to conduct the eQTL analysis by the $\mathrm{R}$ package 'Matrix eQTL' [39] as described in the previous step.

2.3. Differential Expression Analysis of DDT between Genders. According to a recent study, Benedek et al. observed that DDT expression level is significantly higher in brain (white matter) of male MS subjects compared with female MS subjects [15]. However, previous studies reported that the sexspecific expression has already existed in many human genes, including some MS-related genes, for the normal individuals $[45,46]$. Therefore, we further explored whether the sexspecific expression of DDT is a general genetic model for healthy people or associated with MS. Braineac database provided the DDT expression data of 10 brain regions from 134 individuals free of known neurological diseases with the age and age and gender details [34]. We used these data to detect if there is significantly different expression of $D D T$ between genders in the normal individuals by Student's $t$-test (the significance level was set at $P<0.05$ ).

2.4. Effect of rs755622 Polymorphism on DDT Expression Level in Different Gender. To further explore whether the effect of rs755622 polymorphism on DDT expression level has a sex-specific pattern, we selected the rs755622 polymorphism genotype data of 99 male and 35 female subjects from Braineac database [34], respectively. The HWE test of rs755622 polymorphism in male and female groups was performed, respectively, using the $\mathrm{R}$ package 'Genetics' as described in the previous step. And then, we used Fisher's exact test to compare the genotype frequencies of rs755622 minor allele variant $(\mathrm{C})$ between genders by the $\mathrm{R}$ program (http://www.r-project.org/). Finally, in combination with the $D D T$ expression data in the 10 brain regions, we performed the eQTL analysis to assess the influence of rs755622 variant on expression of DDT in males and females, respectively, by the R package 'Matrix eQTL' [39].

\section{Results}

3.1. eQTL Analysis Using Five Large-Scale Datasets. Using the large-scale eQTL data from Braineac, GTEx, CAGE, BIOS QTL Browser, and FHS_eQTL, we validated the effect of rs755622 polymorphism on DDT expression level. 
TABLE 1: Polymorphism rs755622 C allele upregulates DDT expression in brain and blood for subjects without MS diagnosis.

\begin{tabular}{|c|c|c|c|c|c|c|c|}
\hline Gene & SNP & Effect allele & $\mathrm{P}$ value & Effect size $(\beta)$ & Tissues & Sample size & Dataset \\
\hline$D D T$ & rs755622 & $\mathrm{C}$ & $1.47 \mathrm{E}-02 *$ & 0.1282 & Brain intralobular white matter & 134 & Braineac \\
\hline$D D T$ & rs755622 & $\mathrm{C}$ & $3.97 \mathrm{E}-02 *$ & 0.1013 & Brain hippocampus & 134 & Braineac \\
\hline$D D T$ & rs755622 & $\mathrm{C}$ & $1.66 \mathrm{E}-01$ & -0.0874 & Brain cerebellar cortex & 134 & Braineac \\
\hline$D D T$ & rs755622 & $\mathrm{C}$ & $5.84 \mathrm{E}-01$ & 0.0284 & Brain frontal cortex & 134 & Braineac \\
\hline$D D T$ & rs755622 & $\mathrm{C}$ & $3.61 \mathrm{E}-01$ & -0.0436 & Brain medulla & 134 & Braineac \\
\hline$D D T$ & rs755622 & $\mathrm{C}$ & $4.27 \mathrm{E}-01$ & -0.0432 & Brain occipital cortex & 134 & Braineac \\
\hline$D D T$ & rs755622 & $\mathrm{C}$ & $9.63 \mathrm{E}-01$ & -0.0027 & Brain putamen & 134 & Braineac \\
\hline$D D T$ & rs755622 & $\mathrm{C}$ & $2.09 \mathrm{E}-01$ & -0.0630 & Brain substantia nigra & 134 & Braineac \\
\hline$D D T$ & rs755622 & $\mathrm{C}$ & $6.74 \mathrm{E}-01$ & 0.0219 & Brain temporal cortex & 134 & Braineac \\
\hline$D D T$ & rs755622 & $\mathrm{C}$ & $3.87 \mathrm{E}-01$ & -0.0462 & Brain thalamus & 134 & Braineac \\
\hline$D D T$ & rs755622 & $\mathrm{C}$ & $5.09 \mathrm{E}-01$ & -0.0743 & Brain amygdala & 88 & GTEx \\
\hline$D D T$ & rs755622 & $\mathrm{C}$ & $3.28 \mathrm{E}-01$ & -0.1050 & Brain anterior cingulate cortex & 109 & GTEx \\
\hline$D D T$ & rs755622 & $\mathrm{C}$ & $8.55 \mathrm{E}-02$ & 0.1732 & Brain caudate & 144 & GTEx \\
\hline DDT & rs755622 & $\mathrm{C}$ & $3.16 \mathrm{E}-01$ & 0.1241 & Brain cortex & 136 & GTEx \\
\hline$D D T$ & rs755622 & $\mathrm{C}$ & $1.61 \mathrm{E}-01$ & 0.1353 & Brain frontal cortex & 118 & GTEx \\
\hline$D D T$ & rs755622 & $\mathrm{C}$ & 7.21E-01 & -0.0383 & Brain hippocampus & 111 & GTEx \\
\hline$D D T$ & rs755622 & $\mathrm{C}$ & $2.84 \mathrm{E}-01$ & 0.1136 & Brain nucleus accumbens & 130 & GTEx \\
\hline$D D T$ & rs755622 & $\mathrm{C}$ & 7.33E-01 & -0.0417 & Brain putamen & 111 & GTEx \\
\hline$D D T$ & rs755622 & $\mathrm{C}$ & $1.24 \mathrm{E}-01$ & 0.2706 & Brain spinal cord & 83 & GTEx \\
\hline$D D T$ & rs755622 & $\mathrm{C}$ & 7.01E-01 & -0.0553 & Brain substantia nigra & 80 & GTEx \\
\hline$D D T$ & rs755622 & $\mathrm{C}$ & $1.21 \mathrm{E}-04 *$ & 0.5072 & Brain cerebellum & 154 & GTEx \\
\hline$D D T$ & rs755622 & $\mathrm{C}$ & $1.21 \mathrm{E}-03 *$ & 0.3480 & Brain cerebellar hemisphere & 125 & GTEx \\
\hline$D D T$ & rs755622 & $\mathrm{C}$ & $2.91 \mathrm{E}-02 *$ & 0.2388 & Brain hypothalamus & 108 & GTEx \\
\hline$D D T$ & rs755622 & $\mathrm{C}$ & $4.62 \mathrm{E}-06 *$ & 0.2260 & Blood & 369 & GTEx \\
\hline$D D T$ & rs755622 & $\mathrm{C}$ & $3.70 \mathrm{E}-35 *$ & 0.4214 & Blood & 2,765 & CAGE \\
\hline$D D T$ & rs755622 & $\mathrm{C}$ & $4.31 \mathrm{E}-28 *$ & $>0(\mathrm{z}=10.989)$ & Blood & 2,116 & BIOS QTL Browser \\
\hline$D D T$ & rs755622 & $\mathrm{C}$ & $5.21 \mathrm{E}-16 *$ & 0.0427 & Blood & 5,257 & FHS_eQTL \\
\hline
\end{tabular}

The rs755622 position (hg19), 22_24236392_G_C_b37 (G > C); the threshold of significant association is $0.05 ; \beta>0$ and $\beta<0$ means that this effect allele upregulates and downregulates gene expression, respectively; $\beta=\mathrm{z} \times \mathrm{SE}$ (standard errors); the asterisk $(*)$ means a significant association.

Interestingly, we found a significant association between rs755622 and DDT expression in brain (intralobular white matter, hippocampus, cerebellum, cerebellar hemisphere, and hypothalamus) and blood when the significance level was set at $P<0.05$. In addition, we further found that the minor allele variant $(C)$ of rs755622 can significantly upregulate $D D T$ expression $(\beta>0)$ in all of these tissues. More detailed information is described in Table 1.

3.2. eQTL Analysis of MS Subjects Using the RNA-Seq Data. According to the Ensembl database, there are 7 transcripts for the gene DDT (ENST00000398344.8, ENST00000350608.7, ENST00000404092.5, ENST00000430101.2, ENST00000403754.7, ENST00000428792.1, and ENST00000444947.2) [47]. We downloaded the sequences of these transcripts as reference and calculated their TPM values in the MS patients from 2 GEO datasets using the RNA-seq data and Kallisto software. We found that the expression levels of transcripts ENST00000350608.7 and ENST00000444947.2 are generally higher comparing with the other transcripts. These results are described in Supplementary Table S1 and Table S2.

After the genotype calling of rs755622 polymorphism using these RNA-seq data, we found that there are 2 and
1 minor allele variants (C) of rs755622 in 2 MS patients from GSE100297 and GSE66573, respectively, and in other individuals no variant was detected at this polymorphism (Supplementary Table S3). And then, by setting the significance level at $P<0.05$, we further observed that the genotype distribution of rs755622 polymorphism in the 2 groups of MS patients did not deviate from HWE (Supplementary Table S4).

Next, we performed an eQTL analysis using expression level data of the 7 transcripts for gene $D D T$ in combination with genotyping data of rs755622 polymorphism. The results show that the minor allele variant (C) of rs755622 can significantly upregulate the expression of transcript ENST00000444947.2 in blood and transcript ENST00000428792.1 both in brain (optic chiasm) and blood $(P<0.05$ and $\beta>0)$, which is in accordance with the eQTL results of the individuals without MS as described in the previous step. More detailed information is summarized in Table 2.

3.3. Differential Expression Analysis of DDT between Genders. To explore the association between sex-specific expression of $D D T$ and MS, we compared the DDT expression levels 
TABLE 2: Polymorphism rs755622 C allele upregulates DDT expression in brain and blood for MS patients.

\begin{tabular}{llcccccc}
\hline Transcript ID & Gene & SNP & Effect allele & P value & Effect size $(\beta)$ & Tissues & GEO dataset \\
\hline ENST00000428792.1 & DDT & rs755622 & C & $2.23 \mathrm{E}-308$ & $6.72 \mathrm{E}+03$ & Brain optic chiasm & GSE100297 \\
ENST00000428792.1 & DDT & rs755622 & $\mathrm{C}$ & $2.91 \mathrm{E}-15$ & $3.60 \mathrm{E}+01$ & Blood & GSE66573 \\
ENST00000444947.2 & DDT & rs755622 & $\mathrm{C}$ & $1.62 \mathrm{E}-02$ & $1.23 \mathrm{E}+05$ & Blood & GSE66573 \\
\hline
\end{tabular}

The rs755622 position (hg19), 22_24236392_G_C_b37 (G > C); the threshold of significant association is $0.05 ; \beta>0$ and $\beta<0$ means that this effect allele upregulates and downregulates gene expression, respectively.

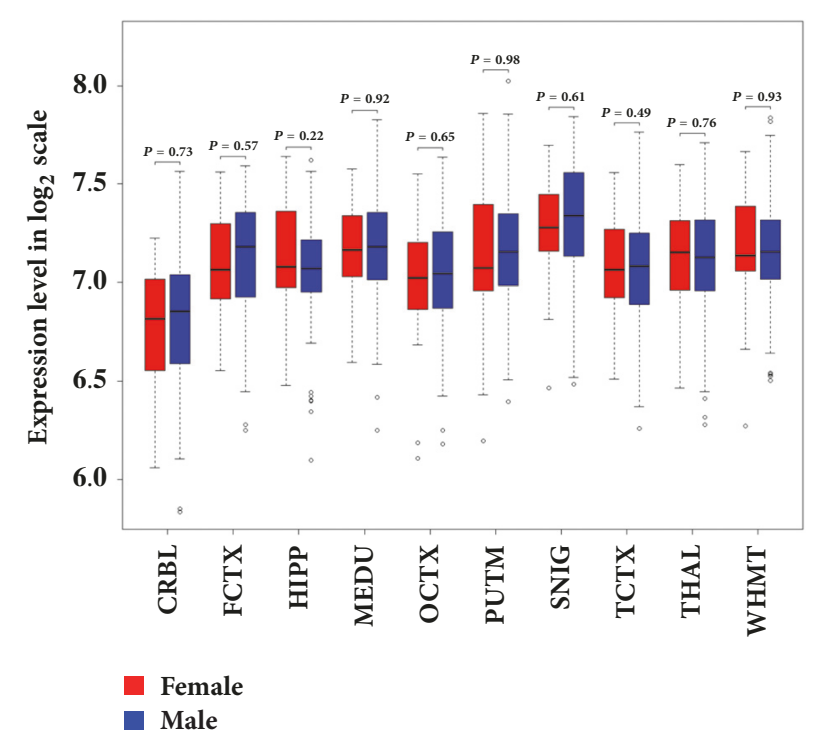

FIGURE 1: Comparison of DDT expression level between healthy male and female subjects in brain. Sample size in these tissues: CRBL (cerebellar cortex): 35 females and 95 males; FCTX (frontal cortex): 34 females and 93 males; HIPP (hippocampus): 30 females and 92 males; MEDU (medulla): 31 females and 88 males; OCTX (occipital cortex): 35 females and 94 males; PUTM (putamen): 33 females and 96 males; SNIG (substantia nigra): 28 females and 73 males; TCTX (temporal cortex): 33 females and 86 males; THAL (thalamus): 33 females and 91 males; WHMT (intralobular white matter): 34 females and 97 males.

between 134 males and females without known neurological diseases in 10 brain regions. The results of Student's $t$-test show that there is no significantly different expression of $D D T$ between normal male and female individuals in all of the 10 brain regions $(P<0.05)$ (Figure 1). Combining Benedek et al.'s findings that $D D T$ is significantly highly expressed in MS males compared to females in brain and its plasma concentration is closely related to the disease severity for MS males but not females [15], we further determined that sexspecific expression of $D D T$ is associated with MS and affects the risk of MS for males.

3.4. Effect of rs755622 Polymorphism on DDT Expression Level in Different Gender. Because the effect of DDT expression on risk of MS has a sex-specific pattern, we further explored whether the regulation of rs755622 polymorphism to $D D T$ expression is also different in different gender. After the HWE test, the genotype distribution of rs755622 polymorphism did not deviate from HWE $(P<0.05)$ both in male and in female groups (Supplementary Table S4). By Fisher's exact test, the frequency of the rs755622 C-containing allele (GC/CC) is no significantly different between genders for healthy subjects $(22.2 \%$ vs. $22.9 \%, P=1)$, while it is significantly higher in male MS subjects compared to female MS subjects according to the results of Benedek et al.s study ( $42.6 \%$ vs. $28.2 \%, P=$ $4.42 \times 10^{-3}$ ) [34]. More detailed information is described in Supplementary Table S5. Finally, the results of eQTL analysis show that the minor allele variant (C) of rs755622 can significantly upregulate $D D T$ expression for males but not females in intralobular white matter and hippocampus $(P<$ 0.05 and $\beta>0$ ). More detailed information is summarized in Table 3.

\section{Discussion}

Both of MIF and DDT, which are highly homologous with each other, play an important role in the modulation of macrophages and microglia immune response $[7,8,11-$ 14]. Previous studies showed that both the MIF expression level and the variant of the rs755622 polymorphism in its promoter region are associated with MS progression [9, $10,16,17]$. Moreover, other studies indicated that rs755622 polymorphism can regulate the expression of MIF gene by affecting the activity of its promoter [18-20]. Recently, Benedek et al. further found that $D D T$ is a sex-specific disease modifier for MS [15]. Given the relationship between MIF and $D D T$, we surmise that rs 755622 polymorphism may also sexspecifically affect the expression of $D D T$ in MS.

We first assessed the influence of rs755622 variant on the expression level of $D D T$ in the normal subjects using five large-scale eQTL datasets [34-38]. And then, we further used two RNA-seq datasets to genotype the rs755622 polymorphism and performed the eQTL analysis to assess this effect in MS patients. Interestingly, the results show that the minor allele variant $(C)$ of rs755622 can significantly upregulate $D D T$ expression level $(P<0.05$ and $\beta>0)$ in brain tissues (intralobular white matter, hippocampus, cerebellum, cerebellar hemisphere, hypothalamus, and optic chiasm) and blood both for normal and MS subjects.

The differential expression analysis shows that there is no significant difference in the expression level of $D D T$ between the healthy male and female subjects. In combination with the results of Benedek et al's study [15], we further determined that the $D D T$ is highly expressed in MS brain tissues and promotes MS progression for males but not females.

Finally, by the eQTL analysis on male and female subjects using Braineac data [34], respectively, we found that 
TABLE 3: Regulation of rs755622 polymorphism to DDT expression level in different gender.

\begin{tabular}{|c|c|c|c|c|c|c|c|}
\hline Gender & Gene & SNP & Effect allele & $P$ value & Effect size $(\beta)$ & Tissues & Sample size \\
\hline Female+Male & $D D T$ & rs755622 & $\mathrm{C}$ & $2.80 \mathrm{E}-02$ & $1.29 \mathrm{E}-01$ & Brain hippocampus & 122 \\
\hline Female & $D D T$ & rs755622 & $\mathrm{C}$ & $1.05 \mathrm{E}-01$ & $1.96 \mathrm{E}-01$ & Brain hippocampus & 30 \\
\hline Male & $D D T$ & rs755622 & $\mathrm{C}$ & 4.41E-02 & $1.40 \mathrm{E}-01$ & Brain hippocampus & 92 \\
\hline Female+Male & $D D T$ & rs755622 & $\mathrm{C}$ & $2.26 \mathrm{E}-02$ & $1.24 \mathrm{E}-01$ & Brain intralobular white matter & 131 \\
\hline Female & $D D T$ & rs755622 & $\mathrm{C}$ & $3.14 \mathrm{E}-01$ & $1.28 \mathrm{E}-01$ & Brain intralobular white matter & 34 \\
\hline Male & $D D T$ & rs755622 & $\mathrm{C}$ & 2.03E-02 & $1.46 \mathrm{E}-01$ & Brain intralobular white matter & 97 \\
\hline
\end{tabular}

Significant associations $(\mathrm{P}<0.05)$ marked in bold. The rs755622 position (hg19), 22_24236392_G_C_b37 (G > C); the threshold of significant association is $0.05 ; \beta>0$ and $\beta<0$ means that this effect allele up-regulates and down-regulates gene expression, respectively.

the minor allele variant $(\mathrm{C})$ of rs755622 can significantly upregulate $D D T$ expression for males but not females. In addition, we also found that the distribution of the rs 755622 polymorphism genotype is not significantly different between genders for healthy subjects, but the frequency of its minor allele variant $(\mathrm{C})$ is significantly higher in male MS subjects according to Benedek et al.s study [15]. Therefore, we think that both the rs 755622 polymorphism sex-specific regulation and its different genotype distribution between genders in MS contribute to the high expression level of $D D T$ in MS males.

In summary, expression level of $D D T$ is significantly high in male MS subjects comparing with females and closely related to the disease severity of MS in males [15]. There is a MS-related SNP rs755622 in the promoter region of MIF which is highly homologous to $D D T$, and the frequency of its minor allele variant $(C)$ is significantly increased and associated with the high expression level of $D D T$ in male MS subjects. These results indicate that the regulation of $D D T$ expression level by the rs 755622 polymorphism can affect MS progression in males, which further supports and expands the findings in previous studies [12-17] and may help to better understand the mechanisms of MS.

\section{Data Availability}

The data used to support the findings of this study are available from the corresponding author upon request.

\section{Conflicts of Interest}

The authors declare that they have no conflicts of interest.

\section{Acknowledgments}

This work was supported by the National Natural Science Foundation of China (no. 31360031); the Major Project of Guizhou Province (Qian Ke He Major Project [2016] 302207); the Youth Science and Technology Talent Project of Guizhou Province [2017] 5617; the Construction Program of Biology First-class Discipline in Guizhou (GNYL [2017] 009).

\section{Supplementary Materials}

Table S1: the expression levels of 7 transcripts for the gene DDT in MS patients from GSE100297. Table S2: the expression levels of 7 transcripts for the gene DDT in MS patients from GSE66573. Table S3: the genotype of rs755622 polymorphism in MS patients from 2 GEO dataset. Table S4: the results of HWE test for genotype distribution of rs755622 polymorphism in different groups. Table S5: the genotype distribution of rs755622 polymorphism between gender in MS and healthy subjects. (Supplementary Materials)

\section{References}

[1] M. Sospedra and R. Martin, "Immunology of multiple sclerosis," Annual Review of Immunology, vol. 23, pp. 683-747, 2005.

[2] E. M. Frohman, M. K. Racke, and C. S. Raine, "Multiple sclerosis-the plaque and its pathogenesis," The New England Journal of Medicine, vol. 354, no. 9, pp. 942-955, 2006.

[3] S.-M. Orton, B. M. Herrera, I. M. Yee et al., "Sex ratio of multiple sclerosis in Canada: a longitudinal study," The Lancet Neurology, vol. 5, no. 11, pp. 932-936, 2006.

[4] P. Browne, D. Chandraratna, C. Angood et al., "Atlas of multiple sclerosis 2013: a growing global problem with widespread inequity," Neurology, vol. 83, no. 11, pp. 1022-1024, 2014.

[5] S. Sawcer, R. J. M. Franklin, and M. Ban, "Multiple sclerosis genetics," The Lancet Neurology, vol. 13, no. 7, pp. 700-709, 2014.

[6] N. A. Patsopoulos, "Genetics of Multiple Sclerosis: An Overview and New Directions," Cold Spring Harbor Perspectives in Medicine, vol. 8, no. 7, 2018.

[7] J. Bauer, I. Huitinga, W. Zhao, H. Lassmann, W. F. Hickey, and C. D. Dijkstra, "The role of macrophages, perivascular cells, and microglial cells in the pathogenesis of experimental autoimmune encephalomyelitis," Glia, vol. 15, no. 4, pp. 437446, 1995.

[8] T. Calandra and T. Roger, "Macrophage migration inhibitory factor: a regulator of innate immunity," Nature Reviews Immunology, vol. 3, no. 10, pp. 791-800, 2003.

[9] G. M. Cox, A. P. Kithcart, D. Pitt et al., "Macrophage migration inhibitory factor potentiates autoimmune-mediated neuroinflammation," The Journal of Immunology, vol. 191, no. 3, pp. 1043-1054, 2013

[10] M. Niino, A. Ogata, S. Kikuchi, K. Tashiro, and J. Nishihira, "Macrophage migration inhibitory factor in the cerebrospinal fluid of patients with conventional and optic-spinal forms of multiple sclerosis and neuro-Behcet's disease," Journal of the Neurological Sciences, vol. 179, no. 1-2, pp. 127-131, 2000.

[11] J. Bernhagen, R. Krohn, H. Lue et al., "MIF is a noncognate ligand of CXC chemokine receptors in inflammatory and atherogenic cell recruitment," Nature Medicine, vol. 13, no. 5, pp. 587-596, 2007. 
[12] N. Esumi, M. Budarf, L. Ciccarelli, B. Sellinger, C. A. Kozak, and G. Wistow, "Conserved gene structure and genomic linkage for D-dopachrome tautomerase (DDT) and MIF," Mammalian Genome, vol. 9, no. 9, pp. 753-757, 1998.

[13] M. Merk, S. Zierow, L. Leng et al., “The D-dopachrome tautomerase (DDT) gene product is a cytokine and functional homolog of macrophage migration inhibitory factor (MIF)," Proceedings of the National Acadamy of Sciences of the United States of America, vol. 108, no. 34, pp. E577-E585, 2011.

[14] M. Merk, R. A. Mitchell, S. Endres, and R. Bucala, "Ddopachrome tautomerase (D-DT or MIF-2): Doubling the MIF cytokine family," Cytokine, vol. 59, no. 1, pp. 10-17, 2012.

[15] G. Benedek, R. Meza-Romero, K. Jordan et al., "MIF and D-DT are potential disease severity modifiers in male MS subjects," Proceedings of the National Acadamy of Sciences of the United States of America, vol. 114, no. 40, pp. E8421-E8429, 2017.

[16] K. Ji, X. Wang, J. Li et al., "Macrophage Migration Inhibitory Factor Polymorphism Is Associated with Susceptibility to Inflammatory Coronary Heart Disease," BioMed Research International, Article ID 315174, 2015.

[17] A. Akcali, S. Pehlivan, M. Pehlivan, T. Sever, and M. Neyal, "Association of macrophage migration inhibitory factor gene promoter polymorphisms with multiple sclerosis in Turkish patients," Journal of International Medical Research, vol. 38, no. 1, pp. 69-77, 2010.

[18] J. A. Baugh, S. Chitnis, S. C. Donnelly et al., "A functional promoter polymorphism in the macrophage migration inhibitory factor (MIF) gene associated with disease severity in rheumatoid arthritis," Genes \& Immunity, vol. 3, no. 3, pp. 170-176, 2002.

[19] B. J. Plant, C. G. Gallagher, R. Bucala et al., "Cystic fibrosis, disease severity, and a macrophage migration inhibitory factor polymorphism," American Journal of Respiratory and Critical Care Medicine, vol. 172, no. 11, pp. 1412-1415, 2005.

[20] S. Yende, D. C. Angus, L. Kong et al., "The influence of macrophage migration inhibitory factor gene polymorphisms on outcome from community-acquired pneumonia," The FASEB Journal, vol. 23, no. 8, pp. 2403-2411, 2009.

[21] X. Bao, G. Liu, Y. Jiang et al., "Cell adhesion molecule pathway genes are regulated by cis-regulatory SNPs and show significantly altered expression in Alzheimer's disease brains," Neurobiology of Aging, vol. 36, no. 10, pp. 2904.el-2904.e7, 2015.

[22] G. Liu, X. Bao, and R. Wang, "Expression quantitative trait loci regulate HNF4A and PTBP1 expression in human brains," Proceedings of the National Acadamy of Sciences of the United States of America, vol. 112, no. 30, p. E3975, 2015.

[23] G. Liu, Y. Hu, S. Jin, F. Zhang, Q. Jiang, and J. Hao, “Cis-eQTLs regulate reduced LST1 gene and NCR3 gene expression and contribute to increased autoimmune disease risk," Proceedings of the National Acadamy of Sciences of the United States of America, vol. 113, no. 42, pp. E6321-E6322, 2016.

[24] G. Liu, Y. Hu, S. Jin, and Q. Jiang, "Genetic variant rs763361 regulates multiple sclerosis CD226 gene expression," Proceedings of the National Acadamy of Sciences of the United States of America, vol. 114, no. 6, pp. E906-E907, 2017.

[25] G. Liu, Y. Liu, Q. Jiang et al., "Convergent Genetic and Expression Datasets Highlight TREM2 in Parkinson's Disease Susceptibility," Molecular Neurobiology, vol. 53, no. 7, pp. 49314938, 2016.

[26] Y. Hu, L. Cheng, Y. Zhang et al., "Rs4878104 contributes to Alzheimer's disease risk and regulates DAPK1 gene expression," Neurological Sciences, vol. 38, no. 7, pp. 1255-1262, 2017.
[27] G. Liu, Y. Zhang, L. Wang et al., "Alzheimer's Disease rs11767557 Variant Regulates EPHA1 Gene Expression Specifically in Human Whole Blood," Journal of Alzheimer's Disease, vol. 61, no. 3, pp. 1077-1088, 2018.

[28] G. Liu, F. Zhang, Y. Jiang et al., "Integrating genome-wide association studies and gene expression data highlights dysregulated multiple sclerosis risk pathways," Multiple Sclerosis Journal, vol. 23, no. 2, pp. 205-212, 2017.

[29] Z. Xiang, M. Xu, M. Liao et al., "Integrating Genome-Wide Association Study and Brain Expression Data Highlights Cell Adhesion Molecules and Purine Metabolism in Alzheimer's Disease," Molecular Neurobiology, vol. 52, no. 1, pp. 514-521, 2015.

[30] G. Liu, Y. Xu, Y. Jiang, L. Zhang, R. Feng, and Q. Jiang, "PICALM rs3851179 variant confers susceptibility to alzheimer's disease in chinese population," Molecular Neurobiology, vol. 54, no. 5, pp. 3131-3136, 2017.

[31] G. Liu, F. Zhang, Y. Hu et al., "Genetic Variants and Multiple Sclerosis Risk Gene SLC9A9 Expression in Distinct Human Brain Regions," Molecular Neurobiology, vol. 54, no. 9, pp. 68206826, 2017.

[32] Y. Zhang, L. Wang, H. Jia et al., "Genetic variants regulate NR1H3 expression and contribute to multiple sclerosis risk," Journal of the Neurological Sciences, vol. 390, pp. 162-165, 2018.

[33] Y. Hu, S. Jin, L. Cheng, G. Liu, and Q. Jiang, "Autoimmune disease variants regulate GSDMB gene expression in human immune cells and whole blood," Proceedings of the National Acadamy of Sciences of the United States of America, vol. 114, no. 38, pp. E7860-E7862, 2017.

[34] A. Ramasamy, D. Trabzuni, S. Guelfi et al., "Genetic variability in the regulation of gene expression in ten regions of the human brain," Nature Neuroscience, vol. 17, no. 10, pp. 1418-1428, 2014.

[35] G. T. Consortium, "Human genomics. The Genotype-Tissue Expression (GTEx) pilot analysis: multitissue gene regulation in humans," Science, vol. 348, no. 6235, pp. 648-660, 2015.

[36] L. R. Lloyd-Jones, A. Holloway, A. McRae et al., "The Genetic Architecture of Gene Expression in Peripheral Blood," American Journal of Human Genetics, vol. 100, no. 2, pp. 228-237, 2017.

[37] D. V. Zhernakova, P. Deelen, M. Vermaat et al., "Identification of context-dependent expression quantitative trait loci in whole blood," Nature Genetics, vol. 49, no. 1, pp. 139-145, 2017.

[38] R. Joehanes, X. Zhang, T. Huan et al., "Integrated genome-wide analysis of expression quantitative trait loci aids interpretation of genomic association studies," Genome Biology, vol. 18, no. 1, p. 16, 2017.

[39] A. A. Shabalin, "Matrix eQTL: Ultra fast eQTL analysis via large matrix operations," Bioinformatics, vol. 28, no. 10, pp. 1353-1358, 2012.

[40] N. Itoh, Y. Itoh, A. Tassoni et al., "Cell-specific and regionspecific transcriptomics in the multiple sclerosis model: Focus on astrocytes," Proceedings of the National Acadamy of Sciences of the United States of America, vol. 115, no. 2, pp. E302-E309, 2018.

[41] C. F. Spurlock 3rd, J. T. Tossberg, Y. Guo, S. Sriram, P. S. Crooke 3rd, and T. M. Aune, "Defective structural RNA processing in relapsing-remitting multiple sclerosis," Genome Biology, vol. 16, no. 1, p. 58, 2015.

[42] N. L. Bray, H. Pimentel, P. Melsted, and L. Pachter, "Nearoptimal probabilistic RNA-seq quantification," Nature Biotechnology, vol. 34, no. 5, pp. 525-527, 2016. 
[43] H. Li and R. Durbin, "Fast and accurate short read alignment with Burrows-Wheeler transform," Bioinformatics, vol. 25, no. 14, pp. 1754-1760, 2009.

[44] H. Li, B. Handsaker, A. Wysoker et al., "The sequence alignment/map format and SAMtools," Bioinformatics, vol. 25, no. 16, pp. 2078-2079, 2009.

[45] C. Ober, D. A. Loisel, and Y. Gilad, "Sex-specific genetic architecture of human disease," Nature Reviews Genetics, vol. 9, no. 12, pp. 911-922, 2008.

[46] K. R. Kukurba, P. Parsana, B. Balliu et al., "Impact of the $\mathrm{X}$ chromosome and sex on regulatory variation," Genome Research, vol. 26, no. 6, pp. 768-777, 2016.

[47] B. L. Aken, P. Achuthan, W. Akanni et al., "Ensembl 2017," Nucleic Acids Research, vol. 45, no. D1, pp. D635-D642, 2017. 


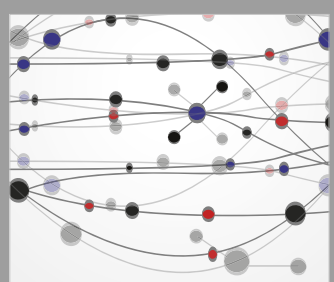

The Scientific World Journal
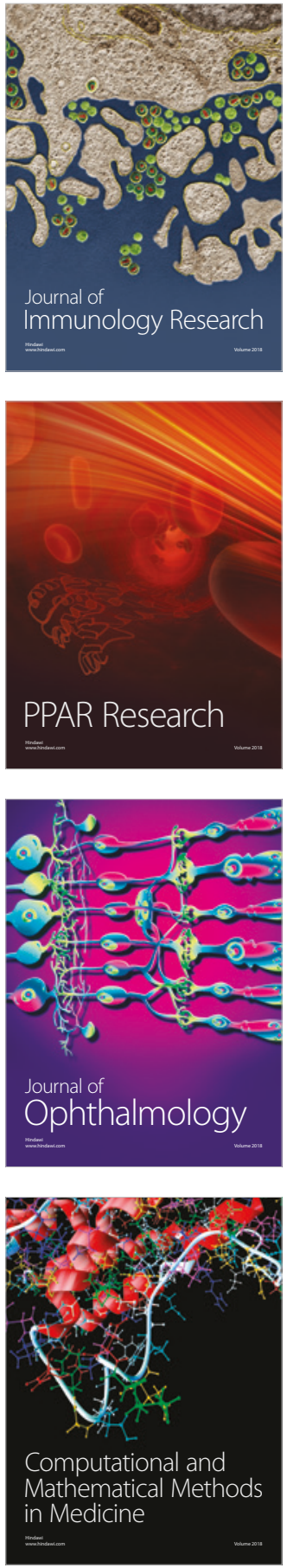

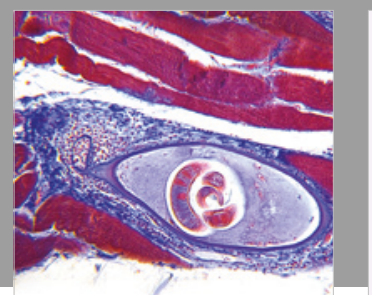

Gastroenterology Research and Practice

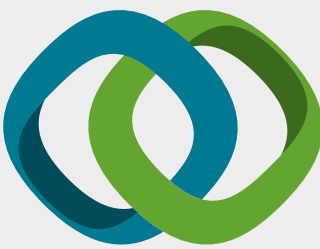

\section{Hindawi}

Submit your manuscripts at

www.hindawi.com
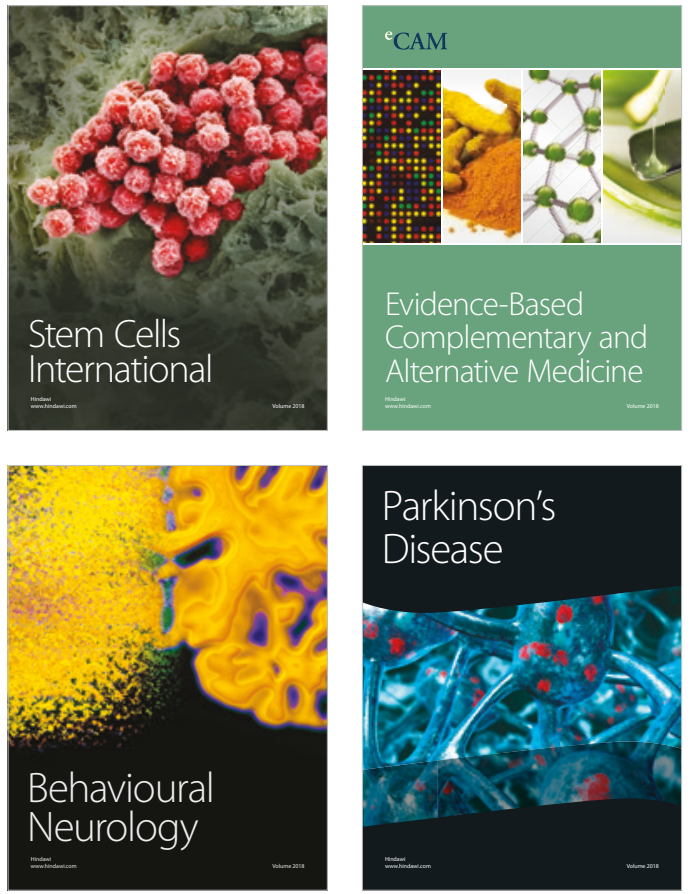

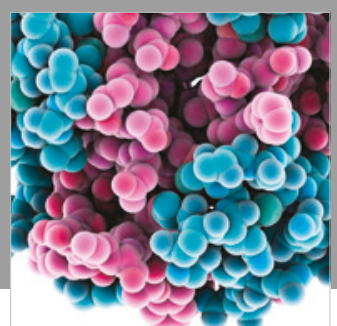

ournal of

Diabetes Research

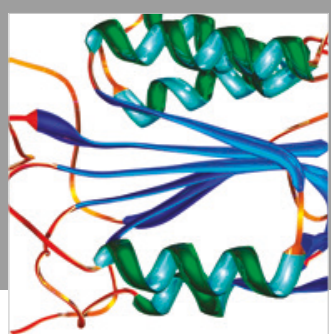

Disease Markers
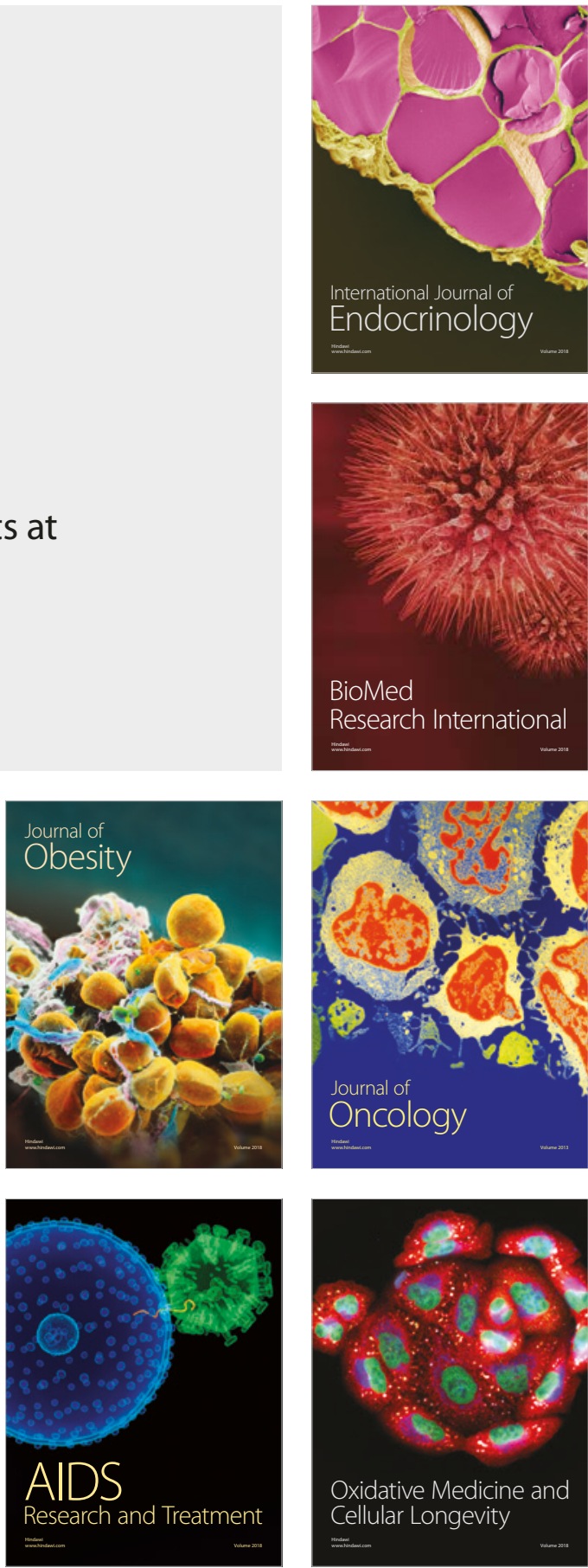